\title{
Parietal involvement in the semantic variant of primary progressive aphasia with Alzheimer's disease CSF profile
}

Géraldine Bera*1,2, Raffaella Migliaccio*2,3 ${ }^{*}$ Thibaut Michelin ${ }^{1}$, Foudil Lamari ${ }^{4}$, Sophie Ferrieux $^{3}$, Marie Nogues ${ }^{3}$, Hugo Bertin ${ }^{5}$, Marie Odile Habert ${ }^{1,5,6}$, Bruno Dubois ${ }^{2,3}$, Marc Teichmann ${ }^{2,3}$, and Aurélie Kas ${ }^{1,5,6}$.

1 Service de Médecine Nucléaire, Groupe Hospitalier Pitié Salpêtrière-Charles Foix, AP-HP, 47-83 Boulevard de l’Hôpital 75651 Paris CEDEX 13, France

2 INSERM U 1127, CNRS UMR 7225, Sorbonne Universités, and Université Pierre et Marie Curie-Paris 6, UMR S 1127, Institut du Cerveau et de la Moelle épinière (ICM), FrontLab, 47-83 Boulevard de l’Hôpital 75651 Paris CEDEX 13, France

3 Département de Neurologie, Institut de la mémoire et de la maladie d’Alzheimer, Groupe Hospitalier Pitié Salpêtrière-Charles Foix, AP-HP, 47-83 Boulevard de l’Hôpital 75651 Paris CEDEX 13 France

4 Laboratoire de Biochimie, AP-HP, Groupe Hospitalier Pitié Salpêtrière-Charles Foix, 47-83 Boulevard de l’Hôpital 75651 Paris CEDEX 13 France

5 http://www.cati-neuroimaging.com

6 Laboratoire d’Imagerie Biomédicale, INSERM U1146, Sorbonne Universités et Université Pierre et Marie Curie-Paris 6, Paris, France

Running title: semantic progressive aphasia with AD biology

\section{Corresponding author:}

Raffaella Migliaccio, Institut du cerveau et de la Moelle Epinière (ICM), FrontLAB, Inserm Unit 1127, 47-83 Bd de l’hôpital, 75651, Paris CEDEX 13, France

Email: lara.migliaccio@gmail.com or lara.migliaccio@icm-institute.org 
Telephone: +33 (0) 157274146

Text word count: 2482

\section{Authors' contributions}

*These authors equally contribute to this work.

Géraldine Bera*: acquisition, analysis and interpretation of imaging data, writing and revising the manuscript. berageraldine@gmail.com

Raffaella Migliaccio*: analysis and interpretation of clinical and imaging data, writing and revising the manuscript. lara.migliaccio@gmail.com

Thibaut Michelin: analysis and interpretation of imaging data. thibautmichelin@gmail.com

Foudil Lamari: analysis and interpretation of CSF biomarkers, revising the manuscript. foudil.lamari@aphp.fr

Marie Odile Habert: acquisition, analysis and interpretation of imaging data, revising the manuscript. marie-odile.habert@aphp.fr

Hugo Bertin: analysis of imaging data. hbertin@imed.jussieu.fr

Sophie Ferrieux and Marie Nogues: acquisition and analysis of neuropsychological data.sophie.ferrieux@aphp.fr; marie.nogues@aphp.fr

Bruno Dubois: acquisition of clinical data, revising the manuscript. bruno.dubois@aphp.fr

Marc Teichmann: acquisition and analysis of clinical data, revising the manuscript. marc.teichmann@aphp.fr

Aurélie Kas: study concept and design, analysis and interpretation of imaging data, writing/revising the manuscript. aurelie.kas@gmail.com 


\begin{abstract}
Semantic variant of primary progressive aphasia (svPPA) is typically associated with non-Alzheimer's disease (AD) pathology. However, some anatomopathological studies have found $\mathrm{AD}$ lesions in those patients. We compared brain perfusion SPECT of 18 svPPA patients with CSF biomarkers indicative of non-Alzheimer pathology (svPPA-nonAD) and three svPPA patients with CSF biomarkers indicative of underlying AD (svPPA-AD). All svPPA patients had severe left temporopolar hypoperfusion. SvPPA-nonAD had additional anterior cingulate and mediofrontal hypoperfusion, whereas svPPA-AD had greater left parietal and posterior cingulate involvement. Parietal damage in svPPA constitutes a biomarker for underlying Alzheimer pathology thus refining the classification of this PPA variant.
\end{abstract}

Keywords: semantic variant of primary progressive aphasia (svPPA), Alzheimer's disease (AD), atypical AD, Single Photon Emission Computed Tomography (SPECT), cerebrospinal-fluid (CSF) examination, parietal lobe. 


\section{INTRODUCTION}

The semantic variant of primary progressive aphasia (svPPA) is characterized by progressive and multimodal loss of semantic knowledge. Patients mainly present with anomia and impaired comprehension of single words. Anatomically, the rostral temporal area show bilateral damage, with left hemispheric predominance [22]. This relatively focal damage makes imaging a useful tool for the diagnostic of svPPA. Pathologically, svPPA is nearly always associated with underlying TAR DNA-binding protein 43 (TDP-43) aggregates $[14,15,23]$. However, in a small proportion of cases, in vivo studies such as cerebrospinal fluid (CSF) analyses and amyloid imaging [19,24-27] and also post mortem series [7-15,28,29] have reported Alzheimer's disease (AD) as pathological substrate (Table 1).

In this context, the biological surrogates for underlying $\mathrm{AD}$ pathology, including amyloid positron emission tomography (PET) and measurements of total tau, phosphorylated tau at threonine 181 (P-tau) and amyloid- $\beta$ peptide $1-42\left(\mathrm{~A} \beta_{42}\right)$ in the CSF, are relevant to define the causative pathology in complex clinical syndromes [24].

Despite a growing number of evidence, little is known about the in vivo imaging pattern of brain damage in svPPA patients with AD pathology, as illustrated in Table 1.

The aim of present work is to investigate brain perfusion patterns in svPPA according to the CSF-AD biomarker profile. We hypothesized that 1) in line with the literature, we would find a small proportion of svPPA patients with a positive AD-CSF profile (svPPA-AD); 2) that the brain hypoperfusion pattern would be different in svPPA-AD, from those having a non-AD CSF biomarker profile (svPPA-nonAD); 3) that in svPPA-AD, the hypoperfusion pattern would involve regions typically and differently damaged in both svPPA and typical amnesic AD. 


\section{METHODS}

\section{Participants and CSF analysis}

We investigated 21 svPPA patients who i) satisfied the international diagnostic criteria of svPPA [22], ii) had a CSF-AD biomarker exploration, and iii) had a brain perfusion single photon emission computed tomography (SPECT). The interval between clinical, biological and imaging evaluations did not exceed six months. For the purpose of image analysis, 24 matched healthy controls, and 24 matched typical amnesic AD patients according to the International Working Group (IWG) [1] were included (for more details see Kas et al. [17] and Table 2). According to French legislation, explicit informed consent was waived. Regulations concerning electronic filing were followed, and patients and their relatives were informed that anonymized data might be used in research investigations.

Quantification of total tau, P-tau and $A \beta_{1-42}$ were performed to define svPPA-AD and svPPA-nonAD subgroups [6]. Diagnosis of $\mathrm{AD}$ has been done on the basis of current biological criteria based on CSF analyses. $A \beta_{42}$ and tau (T-tau or P-tau) are used in combination, and the CSF AD signature, which combines low $\mathrm{A} \beta_{42}$ and high T-tau or P-tau concentrations, significantly increases the accuracy of $\mathrm{AD}$ diagnosis even at a prodromal stage [1]. Ratios are also considered. With respect to biomarker values predictive of AD, a previous study of our department has provided two main cut-offs, namely a P-tau/A $\beta_{42}<0.211$ and a tau $/ \mathrm{A} \beta_{42}<1.23$ [6]; see also for a recent application Teichmann $\mathrm{M}$ et al. [20]. These cutoffs had respective sensitivities of $91.7 \%$ and $95 \%$, and respective specificities of $89.1 \%$ and 84.8\% in distinguishing $\mathrm{AD}$ from other neurodegenerative dementias. Moreover, the ratio of $\operatorname{tau} / \mathrm{A} \beta_{42}$ was the best biomarker to differentiate $\mathrm{AD}$ from frontotemporal lobar degeneration, and showed a specificity of $96.6 \%$ in a series of patients with diagnostic confirmation either by genetics or by post-mortem examination [21]. 


\section{Language and general cognitive assessment}

The language assessment was composed as follows: 1) a picture naming test DO 80 [30]; 2) a single-word comprehension task (requiring pointing to pictures upon auditory word presentation from the Boston Diagnostic Aphasia Evaluation (BDAE) [31]; 3) a sentence repetition task from the BDAE; 4) the Pyramids and Palm Trees Test (PPT), assessing semantic matching [32]. We also evaluated syntax, phonological encoding and motor speech during spontaneous speech and during the description of complex scenes such as 'cookie theft' picture [33]. Global cognitive assessment included the Mini-Mental State Examination [34] and the Frontal Assessment Battery [35]. Non-parametric Mann-Whitney U-test was applied to assess differences among groups for continuous variables. In order to verify the presence of a size effect (e.g. 18 svPPA-nonAD vs. 3 svPPA-AD), a Cohen's test was applied.

\section{Brain perfusion SPECT}

All the patients with svPPA underwent a brain perfusion SPECT with ${ }^{99 \mathrm{~m}} \mathrm{Tc}$ ethylcysteinate dimer $\left({ }^{99 m}\right.$ Tc-ECD). The images were acquired on a three-headed gamma camera equipped with parallel high-resolution collimators (Irix, Philips) allowing a radius of gyration of less $12-13 \mathrm{~cm}$. Projections were reconstructed using an iterative algorithm, postfiltered (low pass filter: order $=4$, cut-off frequency $=0.4 \mathrm{~cm}^{-1}$ ). Reconstructed volumes were spatially normalized into the Montreal Neurological Institute space (MNI) with Statistical Parametric Mapping software (SPM8, Functional Imaging Laboratory, Wellcome Department of Imaging Neuroscience, University College London, London, UK), using a SPECT perfusion template as previously described $[17,20]$. Normalized images were smoothed using an isotropic Gaussian kernel of $12 \mathrm{~mm}$. The dimensions of the resulting voxel were 2x2x2 $\mathrm{mm}$. The analyses were performed with a grey matter threshold set at $40 \%$ of the whole brain mean activity. MNI coordinates and anatomical location were obtained using GingerALE 2.3 
and Talairach Software softwares [36]. All Statistical Parametric Mapping T-maps were obtained using a statistical significance threshold of $\mathrm{p}<0.05$ corrected for multiple tests using the family-wise error rate (FWE) method except for the direct comparison between patients. In this case, considering the number of subjects, a threshold of $\mathrm{p}<0.001$ uncorrected was accepted [6]. To decrease the risk of false positive results, only clusters of more 100 voxels were considered. Age was entered as a covariate. As the threshold of $40 \%$ does not exclude all white matter voxels, SPM T maps were finally visualized after applying a grey matter mask segmented from the Automated Anatomical Labeling (AAL) atlas [18]. The following sets of voxel-based analyses were performed: a) svPPA vs. HC to detect the hypoperfusion pattern in the whole group of patients; b) svPPA-AD vs. HC and svPPA-nonAD vs. HC to analyze differences in hypoperfusion patterns between the two subgroups according to the CSF profile; c) svPPA-nonAD vs. svPPA-AD, and d) svPPA-AD vs. typical amnestic AD, to investigate which regions were specific to SvPPA-AD and which regions overlap.

\section{RESULTS}

\section{CSF profiles and cognitive/language data}

In line with the literature, 18 svPPA patients had normal CSF biomarkers, and three patients had a CSF profile indicative of AD (patients XY, MN, and AB, age 79/63/60 years, education level 13/9/12 years, age of symptoms onset 76/56/58 years, disease duration 3/7/2 years, respectively). The two groups did not differ in terms of demographic characteristics and in general cognitive and language scores (Table 3). However, a size effect was found especially for FAB, sentence repetition, single word comprehension tests, and for complexity index of cookie theft. A detailed description of these patients is here embedded as supplementary material. For each patient, we reported: the clinical history, the tests and scores available (in some case only a qualitative appreciation - presence/absence - was available), 
and the CSF values, until the inclusion in the present study. When available, also information on the follow-up are reported. Figure 1 shows their brain SPECT images.

\section{Brain perfusion profile}

Compared with healthy controls, all svPPA patients had a hypoperfusion of the left hemisphere including the temporal pole and the medial temporal area, the insula and the inferior frontal gyrus, the fusiform gyrus, as well as the caudate nucleus ( $p<0.05 \mathrm{FWE}$, Table 4).

Compared with healthy controls, svPPA-nonAD demonstrated a severe hypoperfusion of the left associative temporal cortex mainly involving the pole and the fusiform $(p<0.05$ FWE; Table 4, and Fig. 2A). When lowering the threshold ( $p<0.001$ uncorrected; Table 4, and Fig.2A) moderate hypoperfusion was found also in: superior, middle, and inferior temporal gyri, insula, inferior frontal gyrus, anterior cingulate and caudate nucleus, in the left hemisphere. Small and scattered clusters were also observed in the left inferior parietal cortex ( $p<0.001$ uncorrected; Table 4, and Fig. 2A). Hypoperfusion was found also in the right hemisphere, including the temporal pole, the fusiform gyrus, the inferior temporal gyrus, and the caudate nucleus ( $p<0.001$ uncorrected; Table 4, and Fig. 2A).

Figure 1 shows brain SPECT images of svPPA-AD patients. Voxel-based analysis in svPPA-AD showed a severe hypoperfusion involving not only the left associative temporal cortex (temporal pole and medial temporal area), but also the left inferior parietal cortex ( $p<0.05$ FWE; Table 4, and Fig. 2B). Lowering the threshold ( $p<0.001$ uncorrected; Table 4, and Fig. 2B), we found hypoperfusion of the posterior cingulate cortex, bilaterally $(p<0.001$ uncorrected; Table 4, and Fig. 2B). The direct comparison between the two svPPA subgroups revealed in svPPA-nonAD a deep hypoperfusion in the right anterior cingulate $(p<0.001$ uncorrected; Table 4, and Fig. 2C). In the svPPA-AD subgroup, the analysis revealed a 
prominent hypoperfusion in the bilateral inferior parietal cortices (with a left side predominance), in the left posterior cingulate and left posterior middle temporal gyrus ( $p<0.001$ uncorrected; Table 4, and Fig. 2C). Then, individual adjusted normalized regional activities values were extracted from the eligible clusters in the left inferior parietal cortex and the left temporal pole revealed by SPM, using the MarsBaR software (http://marsbar.sourceforge.net/). The normalized perfusion values (expressed as a percentage of global cerebral blood flow) were compared between svPPA-AD and svPPA-nonAD. Differences were appreciated in svPPA-AD vs. svPPA-nonAD for the left inferior parietal cortex. No difference and equal distribution were found in svPPA-AD vs. svPPA-nonAD for the left temporal pole (supplementary figure 1). In order to verify the presence of a size effect (e.g. 18 svPPA-nonAD vs. 3 svPPA-AD), a Cohen's test was applied also for these results, and a size effect was found for the parietal peak.

Compared with typical amnesic AD, svPPA-AD patients showed a deep hypoperfusion in the left temporal pole ( $p<0.05$ FWE; Table 4, and Fig. 2C), whereas AD patients had prominent hypoperfusion in the right precuneus and inferior parietal lobule $(p<0.05$ FWE; Table 4, not shown).

Compared with controls, typical amnestic AD had a hypoperfusion in the bilateral posterior associative cortex, including the inferior parietal lobule and posterior cingulate ( $p<0.05$ FWE; Table 4, and Fig. 2D), the bilateral fusiform gyrus. Lowering the threshold, we also found the right hippocampus, the left middle frontal gyrus and the bilateral caudate nucleus ( $<<0.001$ uncorrected; Table 4 and Fig. 2D).

\section{DISCUSSION}

We studied the brain hypoperfusion pattern of 21 svPPA patients with CSF biomarkers indicative, or not, of AD pathology. In three patients, the CSF profile was compatible with a 
diagnosis of underlying $\mathrm{AD}$. The cognitive/language profiles in these patients reflected typical svPPA but the brain perfusion SPECT revealed, in addition to the classical rostral temporal hypoperfusion, a major impairment of left associative parietal cortex. This parietal hypoperfusion in SvPPA-AD fitted with the CSF-AD profile, given that damage to the parietal cortex is typically observed in $\mathrm{AD}$ and reported in both in vivo and post mortem studies [3741]. On the contrary, such parietal involvement is very rarely described in the literature of svPPA.

A distinct pattern of regional atrophy has been highlighted in a voxel-basedmorphometry analysis between svPPA associated with either tau-positive or ubiquitin-positive inclusions and those with post mortem diagnosis of $\mathrm{AD}$ [11]. In the three svPPA-AD patients, the authors revealed a left hippocampal and left superior temporal atrophy (but no parietal involvement) whereas the svPPA patients with frontotemporal dementia (FTD) pathology were associated with typical rostral temporal atrophy [11]. Chow and collaborators [12] described four patients with a clinical diagnosis of svPPA, and a post mortem diagnosis of AD. They found a high AD neuropathologic burden in both temporal regions and left inferior parietal areas. Brain perfusion SPECT performed in two of them, revealed parietal involvement in addition to temporal hypoperfusion. The authors stated '...if this had been more apparent on imaging (e.g., SPECT imaging early in course of illness), perhaps AD would have figured larger on the clinical differential diagnosis’.

It remains unclear whether svPPA-AD patients have AD pathology manifesting as a svPPA syndrome or whether they had coincidental age-related amyloid deposition. This second hypothesis would imply that late-onset svPPA might be more frequently associated with amyloid pathology than early-onset svPPA. A recent amyloid PET study in svPPA does not corroborate this hypothesis [24]. Moreover, two of our svPPA-AD patients have a disease onset before the age of 60 years, which cannot be considered as late-onset. One could 
nevertheless speculate on the coexistence of TDP-43 pathology (predominant in svPPA) and AD pathology [42]. However, in a post mortem study, a concomitant TDP-43 and AD pathology is reported in only one PPA patient (out of 16) [42]. The complexity of the relationships between pathology and the clinical phenotype has also recently been highlighted by Mesulam et al. [43] in a 70-year-old woman with svPPA due to TAR TDP-43-associated FTD. This patient had a positive in vivo amyloid PET imaging. However, the concomitant diagnosis of AD pathology was not confirmed at autopsy. The amyloid PET positivity was considered irrelevant considering the patient's age and considering the fact that similar amyloid imaging positivity can be observed in elderly persons without recognizable cognitive abnormalities. In line with our series, FDG-PET performed in this patient - whose final diagnosis was svPPA associated with FTD pathology - depicted a typical rostral temporal hypometabolism but did not reveal any parietal involvement. Finally, the fact that our three svPPA-AD patients exhibit vulnerability of the posterior neural networks, typically involved in $\mathrm{AD}$, suggests genuine $\mathrm{AD}$ pathology. Present svPPA-AD patients may represent an atypical variant of $\mathrm{AD}$. This widens the spectrum of 'atypical AD' currently including biomarkerpositive patients with posterior cortical atrophy, the logopenic variant of PPA, and behavioural/dysexecutive FTD-like phenotypes [44,45]. All these atypical AD variants demonstrate posterior cortical damage centered on the parietal lobes. Patients with AD and atypical presentations are increasingly identified thanks to CSF-AD biomarkers and molecular imaging.

This study has as major limitation that is the small sample size of svPPA-AD. However, svPPA patients with biological profile of AD are very rare. The percentage of svPPA-AD in our series (14\%) is absolutely in line with previous studies revealing AD pathology in SvPPA [6-10,13-15,19,24-29] (Table 1). Moreover, we acknowledge that it would be preferable to have FDG-PET data for a better spatial resolution and sensitivity. Nevertheless, SPECT 
images were acquired with a dedicated gamma-camera with 3-headed detectors allowing a small radius of gyration of $12-13 \mathrm{~cm}$ providing a high spatial resolution. Finally, another potential limitation is the comparison of unbalanced samples (e.g. 18 SvPPA-NonAD vs. 3 svPPA-AD), strongly highlighting the need to explore in the future a larger sample of svPPAAD patients.

In summary, our results emphasize the critical role of SPECT imaging in clinical practice. In particular, the presence of a hypoperfusion involving the posterior associative cortex could provide helpful information when clinicians are uncertain whether AD or FTD pathology is associated with a predominant semantic deficits [44]. Our findings suggest that parietal damage in svPPA constitutes a biomarker for underlying Alzheimer pathology thus refining the classification of this PPA variant.

\section{ACKNOWLEDGMENTS}

Raffaella Migliaccio gratefully acknowledges the support of 'France Alzheimer' and 'Philippe Chatrier’ Foundations.

\section{CONFLICT OF INTEREST/DISCLOSURE STATEMENT}

We declare no conflict of interest.

This research did not receive any specific grant from funding agencies in the public, commercial, or not-for-profit sectors.

R. Migliaccio, G. Bera, T. Michelin, F. Lamari, S. Ferrieux, M. Nogues, H. Bertin, M. Teichmann report no disclosures.

A. Kas has received speaker honoraria from GE Healthcare, and Piramal.

M.O. Habert has received speaker honoraria from Lilly, GE Healthcare and Piramal. 
B. Dubois has received consulting fees from Eli Lilly. 
TABLES

Table 1. Literature on svPPA related to Alzheimer's disease.

\begin{tabular}{|c|c|c|c|}
\hline Articles & Pathological AD specimen & SvPPA-AD $^{\circ}$ & $\begin{array}{l}\text { Anatomical patterns of brain damage (atrophy, } \\
\text { hypoperfusion or hypometabolism) in svPPA-AD }\end{array}$ \\
\hline Galton 2000 [7] & Histopathology & 1 & Left temporal atrophy and fronto-temporal hypoperfusion \\
\hline Davies 2005 [8] & Histopathology & 2/18 (11\%) & Left posterior temporal atrophy (post mortem) \\
\hline Grossman 2005 [25] & CSF biomarkers & $2 / 13(15 \%)$ & NA \\
\hline Knibb 2006 [9] & Histopathology & $5 / 15(33 \%)$ & NA \\
\hline Alladi 2007 [10] & Histopathology & $2 / 20(10 \%)$ & NA \\
\hline Rabinovici 2008 [46] & Amyloid-positive PETscan & $1 / 5(20 \%)$ & Left anterior temporal hypometabolism \\
\hline Pereira 2009 [11] & Histopathology & $3 / 11(27 \%)$ & Bilateral temporal lobe and left hippocampal atrophy (MRI) \\
\hline Chow 2010 [12] & Histopathology & 5 & Left temporoparietalhypoperfusion \\
\hline Hodges 2010 [13]* & Histopathology & $3 / 24(12.5 \%)$ & Temporopolar and inferior temporal atrophy (MRI) \\
\hline Leyton 2011 [27] & Amyloid-positive PETscan & $1 / 9(11 \%)$ & NA \\
\hline Cruz de Souza, 2011 [19] & CSF biomarkers & $3 / 19(15.8 \%)$ & NA \\
\hline Kas 2012 [26] & CSF biomarkers & $4 / 13(30.8 \%)$ & NA \\
\hline Mesulam 2014 [14] & Histopathology & $1 / 3(33 \%)$ & NA \\
\hline Chare 2014 [15] & Histopathology & 5/31 (16\%) & NA \\
\hline Brown 2016 [24] & Amyloid-positive PETscan & 2/11 (18\%) & NA \\
\hline Santo-Santos 2018 [6] & Amyloid-positive PETscan & $4 / 28(14 \%)$ & Left temporopolar atrophy (MRI) \\
\hline Beaufils 2018 [29] & Amyloid-positive PETscan & $1 / 3(33 \%)$ & NA \\
\hline
\end{tabular}

${ }^{\circ}$ Data are expressed as number of subjects (and percentages)

*extend to the study of Davies [8] 2005 
Table 2. General characteristics of svPPA patients, AD patients and healthy controls.

\begin{tabular}{lccc}
\hline & svPPA patients & AD patients & Healthy controls \\
\hline Number of participants & 21 & 24 & 24 \\
Women/men & $10 / 11$ & $10 / 14$ & $17 / 7$ \\
Right handed & 17 & 22 & 23 \\
Age (years) & $64.0(7.9)$ & $65.1(11.1)$ & $69(6.9)$ \\
Education level (years) & $11.5(3.2)$ & $9.5(4.8)$ & $10.6(4.1)$ \\
Symptom duration (years) & $3.6(2.6)$ & $3.8(2.1)$ & $\mathrm{NA}$ \\
MMSE (/30) & $21.8(5.3)$ & $19.4(5.0)$ & $28.8(0.7)$ \\
FAB (/18) & $12.5(4.1)$ & $11(3.4)$ & $16.8(1.0)$ \\
\hline
\end{tabular}

Abbreviations: $\mathrm{AD}=$ Alzheimer's disease; MMSE=mini-mental state examination; $\mathrm{FAB}=$ frontal assessment battery; NA=not available; SvPPA= semantic variant of primary progressive aphasia.

Numerical data are expressed as mean (and standard deviation). 
Table 3. Demographics, language, general cognitive assessment and CSF ratios.

\begin{tabular}{|c|c|c|c|}
\hline & svPPA-nonAD & svPPA-AD & $p$-values \\
\hline Number of participants & $18(86 \%)$ & $3(14 \%)$ & - \\
\hline Women/men & $8(44 \%) / 10(56 \%)$ & $2(67 \%) / 1(33 \%)$ & $n s^{*}$ \\
\hline Right handed & $14(78 \%)$ & $3(100 \%)$ & $n s^{*}$ \\
\hline Age, years & $63.5(7.7)$ & $67.3(10.2)$ & $n s^{\circ}$ \\
\hline Education level (years) & $11.6(3.4)$ & $11.3(2.1)$ & $n s^{\circ}$ \\
\hline Symptom duration (years) & $3.7(2.5)$ & $5.3(4.9)$ & $n s^{\circ}$ \\
\hline Age of symptom onset (years) & $59.8(8.7)$ & $62.0(12.5)$ & $n s^{\circ}$ \\
\hline MMSE (/30) & $22.1(5.7)$ & $20.0(1.7)$ & $n s^{\circ}$ \\
\hline $\mathrm{FAB}(/ 18)$ & $13.1(3.4)$ & $9.3(6.7)$ & $n s^{\circ}$ \\
\hline Picture naming (/80) & $30.8(22.1)$ & $31.7(30.2)$ & $n s^{\circ}$ \\
\hline Single-word comprehension (/72) & $52.6(15.1)$ & $42.5(13.3)$ & $n s^{\circ}$ \\
\hline PPT Verbal (/50) & $34.0(12.8)$ & $38.0(4.2)$ & $n s^{\circ}$ \\
\hline PPT Visual (/50) & $32.8(11.1)$ & $37.5(0.7)$ & $n s^{\circ}$ \\
\hline Sentence repetition (/16) & $12.6(3.6)$ & $9.0(3.5)$ & $n s^{\circ}$ \\
\hline Cookie theft complexity index & 89.1\% (14.8) & $66.7 \%(0)$ & $n s^{\circ}$ \\
\hline $\mathrm{P}-\operatorname{tau} / \mathrm{A} \beta_{1-42}$ & $0.08(0.04)$ & $0.59(0.44)$ & $0.03^{\circ}$ \\
\hline $\mathrm{Tau} / \mathrm{A} \beta_{1-42}$ & $0.56(0.25)$ & $3.64(2.14)$ & $0.03^{\circ}$ \\
\hline
\end{tabular}

${ }^{\circ}$ Mann-Whitney U-test; *Chi-square test, $p<0.05$.

Abbreviations: $\mathrm{A} \beta_{1-42}=$ Amyloid 1-42 peptide; $\mathrm{FAB}=$ frontal assessment battery; MMSE $=$ mini-mental state examination; ns= non-significant; PPT= Pyramids and Palm Trees Test; svPPA-nonAD= semantic variant of primary progressive aphasia with negative CSF biomarkers for Alzheimer's disease; P-tau= Phosphorylated tau at threonine 181; svPPA-AD= semantic variant of primary progressive aphasia with positive CSF biomarkers for Alzheimer's disease; Tau= total tau. Categorical data are expressed as number and percentage, numerical data as mean (and standard deviation). 
Table 4. Quantitative statistical parametric mapping results: svPPA, svPPA-nonAD, svPPA$\mathrm{AD}$, and $\mathrm{AD}$.

\begin{tabular}{|c|c|c|c|c|}
\hline & \multicolumn{3}{|c|}{ MNI coordinates } & \\
\hline T-score & $\mathrm{X}$ & $\mathrm{y}$ & $\mathrm{Z}$ & Anatomical labels \\
\hline \multicolumn{5}{|c|}{ All svPPA vs controls } \\
\hline 9.94 & -30 & 10 & -36 & Left middle temporal pole* \\
\hline 11.76 & -36 & -14 & -34 & Left fusiform gyrus* \\
\hline 6.12 & -44 & -16 & 16 & Left rolandic operculum* \\
\hline 5.70 & -54 & 40 & 6 & Left inferior frontal gyrus* \\
\hline 6.99 & -4 & 10 & -2 & Left caudate* \\
\hline \multicolumn{5}{|c|}{ svPPA-nonAD vs controls } \\
\hline 9.78 & -30 & 10 & 36 & Left middle temporal pole* \\
\hline 5.12 & 28 & 8 & -34 & Right middle temporal pole \\
\hline 12.11 & -36 & -14 & -34 & Left fusiform* \\
\hline 5.28 & 30 & -6 & -32 & Right fusiform \\
\hline 4.13 & -66 & -34 & 20 & Left superior temporal gyrus \\
\hline 8.93 & -44 & -10 & -32 & Left inferior temporal gyrus \\
\hline 5.12 & 44 & -10 & -32 & Right inferior temporal gyrus \\
\hline 4.13 & -66 & -34 & 20 & Left superior temporal gyrus \\
\hline 6.15 & -4 & 10 & -2 & Left caudate \\
\hline 4.77 & 8 & 8 & 0 & Right caudate \\
\hline 5.77 & -44 & -14 & 16 & Left rolandic operculum \\
\hline 4.93 & -56 & 38 & 6 & Left inferior frontal gyrus \\
\hline 4.50 & -54 & -42 & 52 & Left inferior parietal lobule \\
\hline 4.17 & -4 & 24 & 16 & Left anterior cingulate \\
\hline \multicolumn{5}{|c|}{ svPPA-AD vs controls } \\
\hline 7.19 & -38 & 14 & -32 & Left temporal pole* \\
\hline 8.14 & -58 & -42 & -12 & Left middle temporal gyrus* \\
\hline 7.44 & -54 & -60 & 25 & Left inferior parietal lobule * \\
\hline 3.44 & -2 & -48 & 32 & Left posterior cingulate gyrus \\
\hline 4.16 & 6 & -48 & 30 & Right posterior cingulate gyrus \\
\hline \multicolumn{5}{|c|}{ svPPA-nonAD vs svPPA-AD } \\
\hline 5.45 & 16 & 44 & 12 & Right anterior cingulate gyrus \\
\hline \multicolumn{5}{|c|}{ svPPA-AD vs svPPA-nonAD } \\
\hline 4.61 & -54 & -60 & 40 & Left inferior parietal lobule \\
\hline 4.61 & 48 & -70 & 40 & Right inferior parietal lobule \\
\hline 4.52 & -44 & -72 & 20 & Left temporal middle gyrus \\
\hline 4.30 & -4 & -48 & 32 & Left posterior cingulate gyrus \\
\hline \multicolumn{5}{|c|}{ AD vs. controls } \\
\hline 8.07 & -34 & -48 & -6 & Left fusiform* \\
\hline 8.62 & 36 & -44 & -6 & Right fusiform* \\
\hline 6.57 & -34 & -54 & 44 & Left inferior parietal lobule* \\
\hline 7.14 & 38 & -52 & 44 & Right inferior parietal lobule* \\
\hline 7.37 & 10 & -46 & 30 & Right posterior cingulate gyrus* \\
\hline 4.80 & 20 & -14 & -24 & Right parahippocampal gyrus \\
\hline 4.28 & -44 & 26 & 48 & Left frontal middle gyrus \\
\hline 3.96 & -4 & 12 & 4 & Left caudate \\
\hline 3.78 & 6 & 12 & 4 & Right caudate \\
\hline \multicolumn{5}{|c|}{ svPPA-AD vs. AD } \\
\hline 8.75 & -46 & 10 & -24 & Left temporal pole* \\
\hline 4.93 & -66 & -52 & -20 & Left inferior temporal gyrus \\
\hline \multicolumn{5}{|c|}{ AD vs. svPPA-AD } \\
\hline 5.23 & 20 & -58 & 36 & Right precuneus \\
\hline 4.98 & 30 & -54 & 42 & Right inferior parietal lobule \\
\hline
\end{tabular}

Results are presented at $p<0.001$ uncorrected; ${ }^{*} p<0.05$ family wise error (FWE). 
Abbreviations: $\mathrm{MNI}=$ Montreal Neurological Institute; svPPA-AD=semantic variant of primary progressive aphasia with positive CSF biomarkers for Alzheimer's disease; svPPA-nonAD=semantic variant of primary progressive aphasia with negative CSF biomarkers for Alzheimer's disease.

\section{FIGURES}

Figure 1. Native brain perfusion SPECT of the svPPA patients related to AD. Axial slices are displayed in radiological convention (right is left).

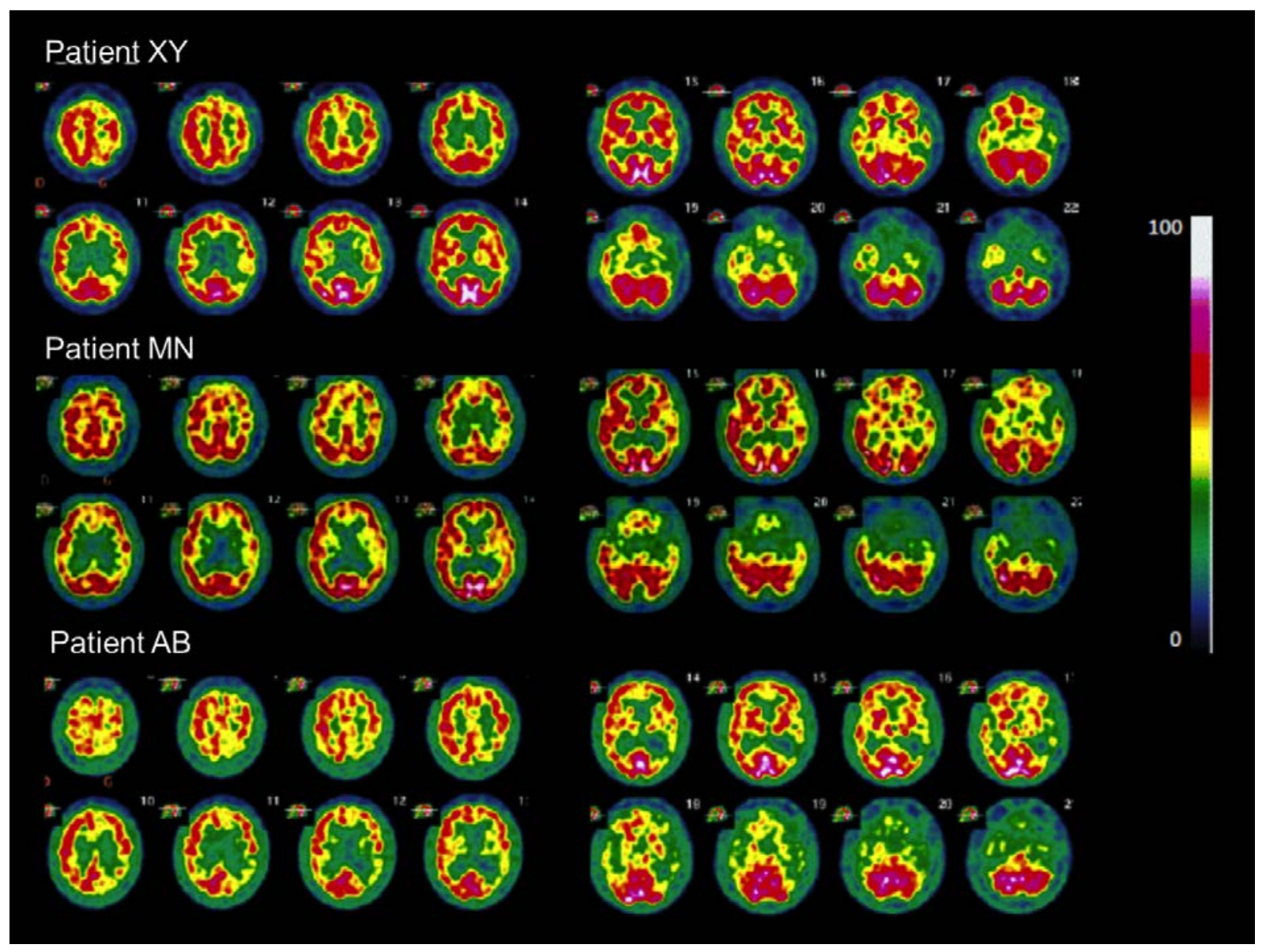

\section{Figure 2. Voxel-based hypoperfusion patterns of svPPA according to CSF profile}

Brain hypoperfusion patterns in semantic variant of primary progressive aphasia (svPPA) patients classified according to their CSF biomarker profiles, with or without Alzheimer's disease (AD), in svPPA-AD or svPPA-nonAD respectively, using Statistical Parametric Mapping (SPM). SPM-T maps are projected onto a surface rendering and onto axial views of the MRI template. The axial slices are shown using neurological conventions ( $\mathrm{R}$ is right, $\mathrm{L}$ is left). The following contrasts are shown: (A) svPPA-nonAD vs. controls; (B) svPPA-AD vs. 
controls; (C) comparison between svPPA-AD, svPPA-nonAD and typical amnestic AD; (D) typical amnestic AD vs. controls.

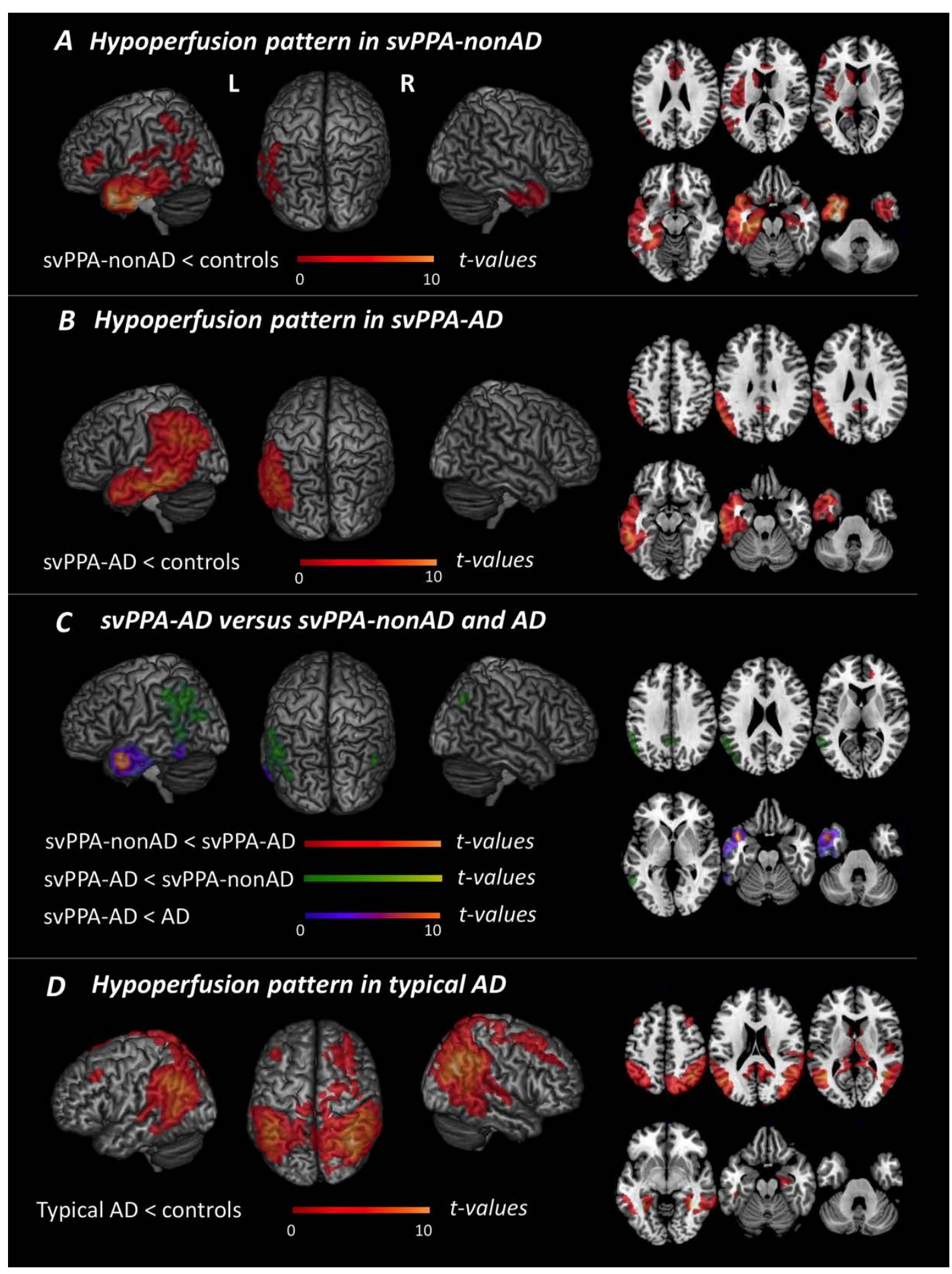




\section{REFERENCES}

[1] Dubois B, Feldman HH, Jacova C, Hampel H, Molinuevo JL, Blennow K, DeKosky ST, Gauthier S, Selkoe D, Bateman R, Cappa S, Crutch S, Engelborghs S, Frisoni GB, Fox NC, Galasko D, Habert M-O, Jicha GA, Nordberg A, Pasquier F, Rabinovici G, Robert P, Rowe C, Salloway S, Sarazin M, Epelbaum S, de Souza LC, Vellas B, Visser PJ, Schneider L, Stern Y, Scheltens P, Cummings JL (2014) Advancing research diagnostic criteria for Alzheimer's disease: the IWG-2 criteria. Lancet Neurol. 13, 614-629.

[2] Gorno-Tempini ML, Murray RC, Rankin KP, Weiner MW, Miller BL (2004) Clinical, cognitive and anatomical evolution from nonfluent progressive aphasia to corticobasal syndrome: a case report. Neurocase 10, 426-436.

[3] Migliaccio R, Agosta F, Toba MN, Samri D, Corlier F, de Souza LC, Chupin M, Sharman M, Gorno-Tempini ML, Dubois B, Filippi M, Bartolomeo P (2012) Brain networks in posterior cortical atrophy: a single case tractography study and literature review. Cortex J. Devoted Study Nerv. Syst. Behav. 48, 1298-1309.

[4] Spinelli EG, Caso F, Agosta F, Gambina G, Magnani G, Canu E, Blasi V, Perani D, Comi G, Falini A, Gorno-Tempini ML, Filippi M (2015) A multimodal neuroimaging study of a case of crossed nonfluent/agrammatic primary progressive aphasia. J. Neurol. 262, 23362345.

[5] Spinelli EG, Mandelli ML, Miller ZA, Santos-Santos MA, Wilson SM, Agosta F, Grinberg LT, Huang EJ, Trojanowski JQ, Meyer M, Henry ML, Comi G, Rabinovici G, Rosen HJ, Filippi M, Miller BL, Seeley WW, Gorno-Tempini ML (2017) Typical and atypical pathology in primary progressive aphasia variants. Ann. Neurol. 81, 430-443.

[6] Santos-Santos MA, Rabinovici GD, Iaccarino L, Ayakta N, Tammewar G, Lobach I, Henry ML, Hubbard I, Mandelli ML, Spinelli E, Miller ZA, Pressman PS, O’Neil JP, Ghosh P, Lazaris A, Meyer M, Watson C, Yoon SJ, Rosen HJ, Grinberg L, Seeley WW, Miller BL, Jagust WJ, Gorno-Tempini ML (2018) Rates of Amyloid Imaging Positivity in Patients With Primary Progressive Aphasia. JAMA Neurol. 75, 342-352.

[7] Galton CJ, Patterson K, Xuereb JH, Hodges JR (2000) Atypical and typical presentations of Alzheimer's disease: a clinical, neuropsychological, neuroimaging and pathological study of 13 cases. Brain J. Neurol. 123 Pt 3, 484-498.

[8] Davies RR, Hodges JR, Kril JJ, Patterson K, Halliday GM, Xuereb JH (2005) The pathological basis of semantic dementia. Brain J. Neurol. 128, 1984-1995.

[9] Knibb JA, Xuereb JH, Patterson K, Hodges JR (2006) Clinical and pathological characterization of progressive aphasia. Ann. Neurol. 59, 156-165.

[10] Alladi S, Xuereb J, Bak T, Nestor P, Knibb J, Patterson K, Hodges JR (2007) Focal cortical presentations of Alzheimer's disease. Brain J. Neurol. 130, 2636-2645.

[11] Pereira JMS, Williams GB, Acosta-Cabronero J, Pengas G, Spillantini MG, Xuereb JH, Hodges JR, Nestor PJ (2009) Atrophy patterns in histologic vs clinical groupings of frontotemporal lobar degeneration. Neurology 72, 1653-1660.

[12] Chow TW, Varpetian A, Moss T, Vinters HV, Marquez S, Miller C (2010) Alzheimer's disease neuropathologic changes in semantic dementia. Neurocase 16, 15-22.

[13] Hodges JR, Mitchell J, Dawson K, Spillantini MG, Xuereb JH, McMonagle P, Nestor PJ, Patterson K (2010) Semantic dementia: demography, familial factors and survival in a consecutive series of 100 cases. Brain J. Neurol. 133, 300-306.

[14] Mesulam M-M, Weintraub S, Rogalski EJ, Wieneke C, Geula C, Bigio EH (2014) Asymmetry and heterogeneity of Alzheimer's and frontotemporal pathology in primary progressive aphasia. Brain 137, 1176-1192.

[15] Chare L, Hodges JR, Leyton CE, McGinley C, Tan RH, Kril JJ, Halliday GM (2014) 
New criteria for frontotemporal dementia syndromes: clinical and pathological diagnostic implications. J. Neurol. Neurosurg. Psychiatry 85, 865-870.

[16] Stamatakis EA, Glabus MF, Wyper DJ, Barnes A, Wilson JT (1999) Validation of statistical parametric mapping (SPM) in assessing cerebral lesions: A simulation study. NeuroImage 10, 397-407.

[17] Kas A, de Souza LC, Samri D, Bartolomeo P, Lacomblez L, Kalafat M, Migliaccio R, Thiebaut de Schotten M, Cohen L, Dubois B, Habert M-O, Sarazin M (2011) Neural correlates of cognitive impairment in posterior cortical atrophy. Brain J. Neurol. 134, 1464 1478.

[18] Tzourio-Mazoyer N, Landeau B, Papathanassiou D, Crivello F, Etard O, Delcroix N, Mazoyer B, Joliot M (2002) Automated anatomical labeling of activations in SPM using a macroscopic anatomical parcellation of the MNI MRI single-subject brain. NeuroImage 15, 273-289.

[19] de Souza LC, Lamari F, Belliard S, Jardel C, Houillier C, De Paz R, Dubois B, Sarazin M (2011) Cerebrospinal fluid biomarkers in the differential diagnosis of Alzheimer's disease from other cortical dementias. J. Neurol. Neurosurg. Psychiatry 82, 240-246.

[20] Teichmann M, Kas A, Boutet C, Ferrieux S, Nogues M, Samri D, Rogan C, Dormont D, Dubois B, Migliaccio R (2013) Deciphering logopenic primary progressive aphasia: a clinical, imaging and biomarker investigation. Brain 136, 3474-3488.

[21] Bian H, Van Swieten JC, Leight S, Massimo L, Wood E, Forman M, Moore P, de Koning I, Clark CM, Rosso S, Trojanowski J, Lee VM-Y, Grossman M (2008) CSF biomarkers in frontotemporal lobar degeneration with known pathology. Neurology 70, 18271835.

[22] Gorno-Tempini ML, Hillis AE, Weintraub S, Kertesz A, Mendez M, Cappa SF, Ogar JM, Rohrer JD, Black S, Boeve BF, Manes F, Dronkers NF, Vandenberghe R, Rascovsky K, Patterson K, Miller BL, Knopman DS, Hodges JR, Mesulam MM, Grossman M (2011) Classification of primary progressive aphasia and its variants. Neurology 76, 1006-1014.

[23] Rohrer JD, Lashley T, Schott JM, Warren JE, Mead S, Isaacs AM, Beck J, Hardy J, de Silva R, Warrington E, Troakes C, Al-Sarraj S, King A, Borroni B, Clarkson MJ, Ourselin S, Holton JL, Fox NC, Revesz T, Rossor MN, Warren JD (2011) Clinical and neuroanatomical signatures of tissue pathology in frontotemporal lobar degeneration. Brain J. Neurol. 134, 2565-2581.

[24] Brown EE, Graff-Guerrero A, Houle S, Mizrahi R, Wilson AA, Pollock BG, Mulsant BH, Felsky D, Voineskos AN, Tang-Wai DF, Verhoeff NPLG, Freedman M, Ismail Z, Chow TW (2016) Amyloid deposition in semantic dementia: a positron emission tomography study: Amyloid- $\beta$ in SD. Int. J. Geriatr. Psychiatry 31, 1064-1074.

[25] Grossman M, Farmer J, Leight S, Work M, Moore P, Van Deerlin V, Pratico D, Clark CM, Coslett HB, Chatterjee A, Gee J, Trojanowski JQ, Lee VM-Y (2005) Cerebrospinal fluid profile in frontotemporal dementia and Alzheimer's disease. Ann. Neurol. 57, 721-729.

[26] Kas A, Uspenskaya O, Lamari F, de Souza LC, Habert M-O, Dubois B, Teichmann M, Sarazin M (2012) Distinct brain perfusion pattern associated with CSF biomarkers profile in primary progressive aphasia. J. Neurol. Neurosurg. Psychiatry 83, 695-698.

[27] Leyton CE, Villemagne VL, Savage S, Pike KE, Ballard KJ, Piguet O, Burrell JR, Rowe CC, Hodges JR (2011) Subtypes of progressive aphasia: application of the international consensus criteria and validation using $\beta$-amyloid imaging. Brain 134, 3030-3043.

[28] Tan RH, Kril JJ, Yang Y, Tom N, Hodges JR, Villemagne VL, Rowe CC, Leyton CE, Kwok JBJ, Ittner LM, Halliday GM (2017) Assessment of amyloid $\beta$ in pathologically confirmed frontotemporal dementia syndromes. Alzheimers Dement. Amst. Neth. 9, 10-20. [29] Beaufils E, Vercouillie J, Vierron E, Cottier J-P, Camus V, Mondon K, Guilloteau D, Hommet C, Ribeiro MJ (2018) Amyloid PET Positivity in Different Primary Progressive 
Aphasia Phenotypes. Clin. Nucl. Med. 43, e103-e108.

[30] Deloche G, Hannequin D (1997) Test de dénomination orale d'images: DO 80, Éd. du Centre de psychologie appliquée, Paris, France.

[31] Goodglass H (2000) Boston Diagnostic Aphasia Examination (BDAE).

[32] Howard D, Patterson KE, Company TVT (1992) The pyramids and palm trees test : a test of semantic access from words and pictures, Bury St Edmunds : Thames Valley Test Company.

[33] Mazaux, Orgogozo J-M (1982) Boston diagnostic aphasia examination. Adaptation française.

[34] Folstein MF, Folstein SE, McHugh PR (1975) "Mini-mental state”. A practical method for grading the cognitive state of patients for the clinician. J. Psychiatr. Res. 12, 189198.

[35] Dubois B, Slachevsky A, Litvan I, Pillon B (2000) The FAB: a Frontal Assessment Battery at bedside. Neurology 55, 1621-1626.

[36] Lancaster JL, Woldorff MG, Parsons LM, Liotti M, Freitas CS, Rainey L, Kochunov PV, Nickerson D, Mikiten SA, Fox PT (2000) Automated Talairach atlas labels for functional brain mapping. Hum. Brain Mapp. 10, 120-131.

[37] Brun A, Englund E (1981) Regional pattern of degeneration in Alzheimer's disease: neuronal loss and histopathological grading. Histopathology 5, 549-564.

[38] Chase TN, Foster NL, Mansi L (1983) Alzheimer's disease and the parietal lobe. Lancet Lond. Engl. 2, 225.

[39] Bloudek LM, Spackman DE, Blankenburg M, Sullivan SD (2011) Review and metaanalysis of biomarkers and diagnostic imaging in Alzheimer's disease. J. Alzheimers Dis.

$J A D$ 26, 627-645.

[40] Foster NL, Chase TN, Mansi L, Brooks R, Fedio P, Patronas NJ, Di Chiro G (1984) Cortical abnormalities in Alzheimer's disease. Ann. Neurol. 16, 649-654.

[41] Bruner E, Jacobs HIL (2013) Alzheimer's disease: the downside of a highly evolved parietal lobe? J. Alzheimers Dis. JAD 35, 227-240.

[42] Bigio EH, Mishra M, Hatanpaa KJ, White CL, Johnson N, Rademaker A, Weitner BB, Deng H-X, Dubner SD, Weintraub S, Mesulam M (2010) TDP-43 pathology in primary progressive aphasia and frontotemporal dementia with pathologic Alzheimer disease. Acta Neuropathol. (Berl.) 120, 43-54.

[43] Mesulam M-M, Dickerson BC, Sherman JC, Hochberg D, Gonzalez RG, Johnson KA, Frosch MP (2017) Case 1-2017: A 70-Year-Old Woman with Gradually Progressive Loss of Language. N. Engl. J. Med. 376, 158-167.

[44] Ossenkoppele R, Pijnenburg YAL, Perry DC, Cohn-Sheehy BI, Scheltens NME, Vogel JW, Kramer JH, van der Vlies AE, Joie RL, Rosen HJ, van der Flier WM, Grinberg LT, Rozemuller AJ, Huang EJ, van Berckel BNM, Miller BL, Barkhof F, Jagust WJ, Scheltens P, Seeley WW, Rabinovici GD (2015) The behavioural/dysexecutive variant of Alzheimer's disease: clinical, neuroimaging and pathological features. Brain 138, 2732-2749. [45] Migliaccio R, Agosta F, Rascovsky K, Karydas A, Bonasera S, Rabinovici GD, Miller BL, Gorno-Tempini ML (2009) Clinical syndromes associated with posterior atrophy: Early age at onset AD spectrum. Neurology 73, 1571-1578.

[46] Rabinovici GD, Jagust WJ, Furst AJ, Ogar JM, Racine CA, Mormino EC, O’Neil JP, Lal RA, Dronkers NF, Miller BL, Gorno-Tempini ML (2008) Abeta amyloid and glucose metabolism in three variants of primary progressive aphasia. Ann. Neurol. 64, 388-401. 


\section{SUPPLEMENTARY MATERIAL}

\section{Clinical cases}

\section{Patient X.Y.}

XY was a 60-year-old right-handed woman, without a family history of dementia. She was first seen at the Pitié-Salpêtrière Hospital in 2008, two years after symptom onset. She started to notice a progressive worsening deficit in retrieving words. XY did not report difficulty recognizing and using objects, nor difficulties identifying people, despite clear deficits in these tasks on testing (see below).

In 2008, at the time of her first visit, her mood was good. Personality was not markedly changed. She was completely independent in her personal needs and still participated in handling their finances. On examination, $\mathrm{XY}$ was completely orientated. She was able to transfer the general message about her current troubles and to report autobiographical events. Her behavior was appropriate but with a discrete indifference and joviality. Her language production was fluent, grammatically correct and her speech was well articulated and fast. However, she presented logorrhea, semantic paraphasias, deficit of comprehension mainly due to the fact that she did not know the meaning of many of the words, verbal perseverations, and surface dyslexia.

General neurological exam was normal. Specific speech and language tests were administered. XY showed a marked deficit on naming (DO 80=72/80 [1]), and especially in confrontation naming (PPTT= 35/50 [2]). Her deficit was not limited to a defective recall of lexical labels, but also extended to general knowledge associated with them. In fact, she was also impaired in a semantic association task involving only pictures (PPTT, 38/50 [2]). XY also showed difficulties in naming (6/35) and recognizing famous faces (29/35). Reading and writing were spared (BDAE [3]). Repetition of single words and simple sentences was intact (BDAE [3]). Patient was mildly impaired in spelling irregular words (7/8) (BDAE [3]). 
Category verbal fluency was impaired (score=4). Neuropsychological evaluation showed a decline in overall cognitive efficiency justified by the severity of the sematic disorders (MMSE score $=22 / 30$ [4]) with a normal temporospatial orientation; working memory disorders (backward verbal span=3 [5]) with spared episodic memory (total recall 45/48, and reactivity to indices 91\% at FCSRT [6]). A 'frontal' evaluation was performed. The TMT [7] showed severe slowness (time of execution part B > 300’ sec), and very poor performances (2/14 correct lines). The performance at Wisconsin card sorting test [8] was poor (number of criteria= 1/6; 73\% of errors; $54 \%$ of perseverations). Her visuospatial skills were intact (copy of Rey figure [9] score=34/36), with good recognition abilities when the distractors had little to do with the target and with very low performance when the distractors were very conceptually close to the target and required an explicit recovery effort of the source of learning. Her praxis functions were intact (16/17), as her calculation skills (clinical assessment).

On the basis of a clinical history of worsening deficit in semantic memory, relative sparing of visuospatial and praxic functions, and development of some behavioral disturbances, as well as the MRI and SPECT brain damage pattern (figure 1, patient XY), a diagnosis of svPPA was established. In particular, the MRI showed marked atrophy of the left anterior temporal lobe with enlargement of the temporal horn of the lateral ventricle. The SPECT showed a severe left temporal pole hypoperfusion including the mesiotemporal regions, an extension to the left parieto-temporal junction and a moderate hypoperfusion of the right temporal pole. At that time, a CSF examination was performed and a biological profile of AD was found $\left(\mathrm{Tau} / \mathrm{A} \beta_{42}=5.5, \mathrm{P}-\mathrm{Tau} / \mathrm{A} \beta_{42}=1.03\right)$.

In 2011, she had a new language assessment showing a marked deficit on naming (DO $80=46 / 80$ [1]). Reading was still spared, but writing became impaired particularly for single low frequency words. However, repetition of single words and simple sentences was still intact. Patient was impaired in spelling regular and irregular words. Category verbal fluency was 
impaired (score=3). She also had a new cognitive evaluation: MMSE score was 7/30 [4], with a strong spatial and temporal disorientation; Mattis Dementia Rating Scale [10] score was 54/144, with poor performances in attention, conceptualization and memory; FAB score was 8/18 [11]; TMT A showed a slowness (124 sec.) [7]; she also had a severe working memory problem (digit span backwards verbal=2; spatial=2), and praxic difficulties, especially for symbolic gestures.

$\mathrm{XY}$ has a long follow-up over the time (6 years) with semantic deficits always present and core of the syndrome, and with a mutism in the last few years.

\section{Patient M.N.}

MN was a 79-year-old right-handed man, without a family history of dementia. He was first seen at the Pitié-Salpêtrière Hospital in 2011, three years after symptom onset. He started to notice a progressive worsening deficit in retrieving words, associated with slight memory problems. At his first visit at the Pitié-Salpêtrière Hospital, MN was described by the son as a very active person, with several interests and hobbies (e.g. gardening), and highly participative to the life of family and still very independent. However, at very first neurological and neuropsychological evaluation, he appeared as depressed.

On examination, MN was well orientated in the time, and somewhat disoriented in the space. He was able to transfer the general message about his current troubles and to report autobiographical events. His behavior was appropriate. General neurological exam was normal. Spontaneous speech was fluent and informative, and the word finding deficit was little noticeable. Language evaluation showed severe perturbations of the semantic system with: loss of meaning, semantic paraphasias, surface dyslexia and dysgraphia, confrontation naming deficits. He had a moderate-to-severe aphasia (BDAE, global score 3/5 [3]), naming deficits (DO 80 24/80 [1]), as well as great confrontation naming difficulties (PPTT, verbal 35/50; 
visual 38/50 [2]). Category verbal fluency was impaired (score=2). MN also showed difficulties in naming (9/35) and recognizing famous faces (0/35). Reading and writing were impaired especially for irregular words (BDAE [3]; irregular words score=1/10). Repetition of sentences was altered (BDAE [3]; score=13/16). Patient was mildly impaired in spelling irregular words (BDAE [3]; score=3/8). Neuropsychological evaluation showed a decline in overall cognitive efficiency justified by the severity of the sematic disorders (MMSE score=21/30 [4]; Mattis Dementia Rating Scale score=118/144 [10]; FAB score=15/18 [11]). Working memory was spared (digit verbal and visual span backward=5 [5]). Visual episodic memory was spared (delayed recall of Rey’s figure=18.5/36 [9]). Verbal episodic memory was not evaluable because of semantic deficits in reading and recognition (FCSRT [6]). His praxis functions were intact, but visuospatial skills were altered (copy of Rey figure [9] score=27/36).

On the basis of a clinical history of worsening deficit in semantic memory, as well as the MRI and SPECT brain damage pattern (figure 1, patient MN), a diagnosis of semantic dementia svPPA was established. In particular, the MRI showed marked atrophy of left temporal (polar and medio-temporal) and insular cortices. The SPECT showed bilateral temporal pole hypoperfusion with left-sided predominance, with extension to the parieto-temporal junction. At that time, a CSF examination was performed and a biological profile of AD was found $\left(\mathrm{Tau} / \mathrm{A} \beta_{42}=1.3, \mathrm{P}-\mathrm{Tau} / \mathrm{A} \beta_{42}=0.16\right)$.

He continued to be seen at the Pitié-Salpêtrière Hospital for almost 5 years, and he showed a progressive worsening not only in semantics, but also in attention and visual memory.

\section{Patient A.B.}

AB was a 63-year-old woman, without a family history of dementia, and presenting with early language difficulties as such errors in giving children's names, started seven years before (2002) to be seen at Pitié-Salpêtrière Hospital (2008). In 2002, she complained a progressive 
appearance of language problems, with clear difficulties in word finding and in some case in word comprehension. At that time, she had a first neurological visit in a French hospital. The report described a MMSE score of 27/30 [4], a score of 76/80 in the naming test (DO 80, [1]), and a severe surface dysgraphia (BDAE [3]; writing score=9/32). Memory (Free and Cued Selective Reminding Test; FCSRT, [6]), praxies (clinical assessment), and visuospatial functioning (Rey’s figure copy [9]) were completely spared. The first cerebral MRI was reported as normal.

In 2004, the patient started experiencing worsening difficulties in naming, and a less detailed evaluation showed a score of 74/80 in the naming test (DO 80 [1]). Episodic verbal memory test (FCSRT [6]) scores were still normal.

In 2005, a cognitive and language follow-up assessment found a score of 25/30 at MMSE [4], of 63/80 at DO 80 [1], clear surface agraphia and dyslexia, slight decline in memory test with a total recall of 41/48, and lower reactivity to indices (61\%) at FCSRT [6]. The medical report mentioned a first brain SPECT showing a left temporal pole hypoperfusion (images not seen).

In 2007, the patient had a pathological score at MMSE (18/30) [4], strong difficulties in naming (DO 80 score= 39/80 [1]), decline in semantic fluency (score=4), and words and short sentences comprehension deficits (BDAE [3]; score=6/10). She also showed worsening writing difficulties (BDAE [3]; writing score=5/26), in particular for irregular words (3/32) compared to regular (18/32). The same trend was evidenced also in reading test (irregular 25/3, regular words 32/34). She performed pathologically in semantic association task (Pyramid and Palm Trees test, [2]). Her deficit was not limited to a defective recall of lexical labels (PPTT written words, 41/50 [2]), but also extended to general knowledge associated with them. In fact, she was also impaired in a semantic association task involving only pictures (PPTT, 37/50 [2]). She also presented memory deficits (total recall=16/48, and reactivity to indices $=18 \%$, but still no 
intrusions at FCSRT [6]). Gestural (clinical assessment) and constructional apraxia (copy of Rey’s figure [9]) were absent. Calculation skills were preserved. Attention was already impaired: the trail making test (TMT, [7]) showed severe slowness (time of execution part B > $600 \mathrm{sec})$.

In 2008, AB was seen for the first time at Pitié-Salpetriere hospital. At that time, the patient mainly presented with difficulties to speak, understand, read, and write. No sleep or appetite disturbances. Moderate autonomy loss. General neurological exam was normal. Language assessments showed: asemantic speech, semantic paraphasias, word finding problem particularly evident with low frequency words. Scores were very low in all the tests examining language abilities. On the basis of a clinical history of worsening deficit in semantic memory, relative sparing of visuospatial functions, and development of some behavioral disturbances (as perseverations), as well as the SPECT hypoperfusion pattern (figure 1, patient AB), a diagnosis of semantic dementia (now called svPPA) was established. In particular, the SPECT showed a severe hypoperfusion of bilateral temporal poles, including the medial temporal structures, and extending to parieto-temporal associative cortices, with great left side predominance. Additional areas of hypoperfusion were also found in mediofrontal cortex, with left side predominance. At that time, a cerebrospinal (CSF) fluid examination was performed and a biological profile of $\mathrm{AD}$ was found $\left(\mathrm{Tau} / \mathrm{A} \beta_{42}=4.1, \mathrm{P}-\mathrm{Tau} / \mathrm{A} \beta_{42}=0.57\right)$. 


\section{SUPPELMENTARY MATERIAL REFERENCES}

[1] Deloche G, Hannequin D (1997) Test de dénomination orale d'images: DO 80, Éd. du Centre de psychologie appliquée, Paris, France.

[2] Howard D, Patterson KE, Company TVT (1992) The pyramids and palm trees test : a test of semantic access from words and pictures, Bury St Edmunds : Thames Valley Test Company.

[3] Goodglass H (2000) Boston Diagnostic Aphasia Examination (BDAE).

[4] Folstein MF, Folstein SE, McHugh PR (1975) "Mini-mental state”. A practical method for grading the cognitive state of patients for the clinician. J. Psychiatr. Res. 12, 189198.

[5] Hilbert S, Nakagawa TT, Puci P, Zech A, Bühner M (2014) The Digit Span Backwards Task. Eur. J. Psychol. Assess. 31, 174-180.

[6] Grober E, Sanders AE, Hall C, Lipton RB (2010) Free and Cued Selective Reminding Identifies Very Mild Dementia in Primary Care. Alzheimer Dis. Assoc. Disord. 24, 284290.

[7] Tombaugh TN (2004) Trail Making Test A and B: Normative data stratified by age and education. Arch. Clin. Neuropsychol. 19, 203-214.

[8] Kohli A, Kaur M (2006) Wisconsin Card Sorting Test: Normative data and experience. Indian J. Psychiatry 48, 181-184.

[9] Rivera D, Perrin PB, Morlett-Paredes A, Galarza-Del-Angel J, Martínez C, Garza MT, Saracho CP, Rodríguez W, Rodríguez-Agudelo Y, Rábago B, Aliaga A, Schebela S, Luna M, Longoni M, Ocampo-Barba N, Fernández E, Esenarro L, García-Egan P, Arango-Lasprilla JC (2015) Rey-Osterrieth Complex Figure - copy and immediate recall: Normative data for the Latin American Spanish speaking adult population. NeuroRehabilitation 37, 677-698.

[10] Foss MP, de Carvalho VA, Machado TH, dos Reis GC, Tumas V, Caramelli P, Nitrini R, Porto CS (2013) Mattis dementia rating scale (DRS) normative data for the brazilian middle-age and elderly populations. Dement. Neuropsychol. 7, 374-379.

[11] Dubois B, Slachevsky A, Litvan I, Pillon B (2000) The FAB: a Frontal Assessment Battery at bedside. Neurology 55, 1621-1626.

\section{SUPPLEMENTARY FIGURE}

\section{Supplementary figure 1: Plots of the normalized perfusion values in the left temporal}

\section{pole and the left inferior parietal cortex in patients and controls.}

Individual adjusted normalized activities values in the left temporal pole and left inferior parietal cortex were extracted from the eligible clusters revealed by Statistical Parametric Mapping (SPM), using the MarsBaR software, and compared among the three groups: semantic variant of primary progressive aphasia, with Alzheimer's disease cerebrospinal fluid profile (svPPA-AD, white circles), without AD (svPPA-nonAD) and healthy volunteers. The 
normalized perfusion values are expressed as a percentage of global cerebral blood flow. Differences were appreciated in svPPA-AD vs. svPPA-nonAD for the left inferior parietal cortex. No difference and equal distribution were found in svPPA-AD vs. svPPA-nonAD for the left temporal pole.

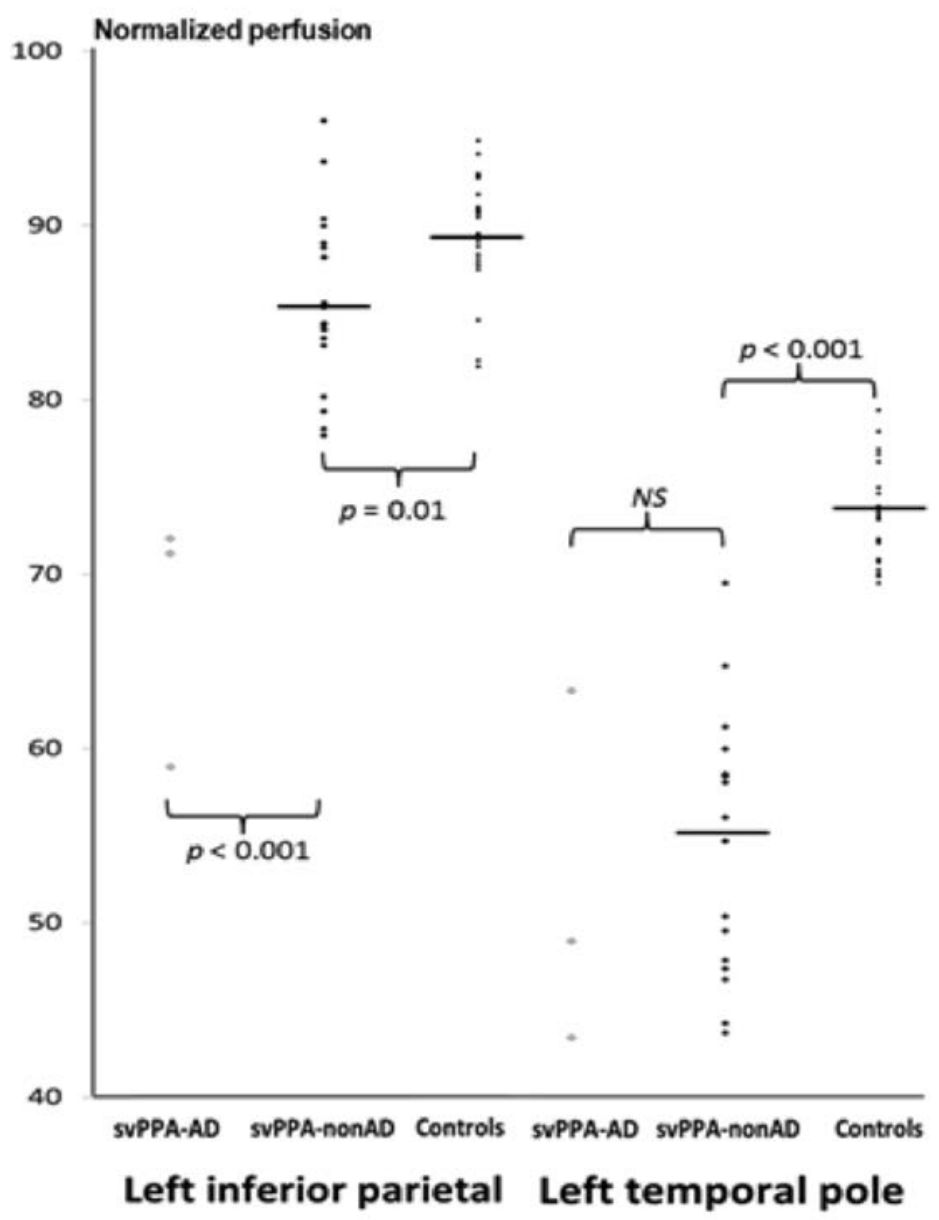

